\title{
Sparen und Investieren im 21. Jahrhundert: Das Ende der Kapitalknappheit
}

Bereits seit längerer Zeit bewegen sich die Realzinsen (Nominalzinsen abzüglich Inflationsrate) in zahlreichen Ländern im Bereich von 0\%. Dafür machen viele die Europäische Zentralbank (EZB) und die von inr verfolgte expansive Geldpolitik verantwortlich. Diese Kritik an der EZB ist jedoch ungerechtfertigt, da die Null-Zinsen auf fundamentale Faktoren auf dem Kapitalmarkt zurückgeführt werden können. In unserem Buch (v. Weizsäcker und Krämer, 2019) kommen wir zu dem Ergebnis, dass es sich hierbei nicht um ein temporäres Phänomen handelt, sondern dass es strukturelle Ursachen gibt, die das Angebot an und die Nachfrage nach Kapital bestimmen. Unsere Analyse zeigt, dass Kapital im 21. Jahrhundert nicht länger knapp und dass der natürliche Zins negativ ist. Diese Erkenntnisse erfordern sowohl ein andersartiges makroökonomisches Denken als auch andere wirtschaftspolitische Maßnahmen als bisher.

Für unsere Untersuchung haben wir auf die in der ökonomischen Wachstums- und Kapitaltheorie bewährte Methode der Steady-State-Analyse zurückgegriffen, die eine lange Tradition in der ökonomischen Theorie hat und bereits von Adam Smith (1776) angewandt wurde. Auf der Grundlage einer Steady-State-Analyse ist es möglich, säkulare Trends zu erkennen. Wir konzentrieren uns auf reale ökonomische Größen. Eine monetäre Analyse ist für unsere Fragestellung weder erforderlich noch sinnvoll. Wir nutzen einen vom bedeutenden österreichischen Ökonomen Eugen von Böhm-Bawerk (1889) Ende des 19. Jahrhunderts in die Literatur eingeführten Zeitbegriff: die „Produktionsperiode“ $(T)$. Hierunter versteht man den durchschnittlichen zeitlichen Abstand zwischen dem ursprünglichen Input (Arbeit und Boden) und dem letztendlichen Output (Konsumgüter). Ihr entspricht approximativ der Kapitalkoeffizient, der hier als das Verhältnis zwischen dem privatwirtschaftlichen Kapitalbestand und dem jährlichen Konsum definiert ist. Dieser ist empirisch leicht zu ermitteln. Einer arbeitsteiligen, durch den massiven Einsatz von Kapitalgütern gekennzeichneten Produktionsweise entspricht eine lange Produktionsperiode. BöhmBawerk entwickelte den anschaulichen Begriff von der „Mehrergiebigkeit längerer Produktionsumwege“. Analog

(C) Der/die Autor(en) 2020. Open Access: Dieser Artikel wird unter der Creative Commons Namensnennung 4.0 International Lizenz (https:// creativecommons.org/licenses/by/4.0/deed.de) veröffentlicht.

Open Access wird durch die ZBW - Leibniz-Informationszentrum Wirtschaft gefördert. zur Produktionsperiode $T$ für den Produktionssektor der Volkswirtschaft haben wir für den Sektor der privaten Haushalte die „Warteperiode" $Z$ entwickelt. Sie ist ebenfalls eine Zeitgröße. Unter der Warteperiode verstehen wir den in Gegenwartswerten ausgedrückten durchschnittlichen zeitlichen Vorlauf der Arbeits- und Bodenentlohnungen vor den mit innen finanzierten Konsumausgaben. In der Zwischenzeit werden diese Entlohnungen gespart. Daher sind die kumulierten Ersparnisse aller privaten Haushalte proportional zu dieser durchschnittlichen Warteperiode. Im Normalfall eines privaten Haushalts ist die Warteperiode positiv. Daher ist auch die volkswirtschaftliche private Warteperiode $Z$ positiv. Schließlich gibt es in unserem Denkansatz noch den Vermögenswert des Bodens. Auch diesen können wir auf den volkswirtschaftlichen Konsum beziehen. Die entsprechende „Bodenperiode" bezeichnen wir mit $L$.

Das Gleichgewicht zwischen dem durch kumulierte Sparleistungen angestrebten Vermögenswunsch $Z$ und dem Realvermögen $T+L$ wird durch den Realzinssatz $(r)$ und die „Staatsschuldenperiode“ $(D)$ hergestellt. Die Staatsschuldenperiode ist der vierte von uns eingeführte Zeitbegriff. Sie baut die Brücke zwischen den drei ersten Zeitgrößen. Die Summe aus der privaten Warteperiode $Z$ und der staatlichen Warteperiode $(-D)$ (den Staatsschulden) ist die gesamte, die volkswirtschaftliche, Warteperiode. Wir leiten her, dass im makroökonomischen Gleichgewicht die volkswirtschaftliche Warteperiode $Z-D$ approximativ gleich der Summe aus der volkswirtschaftlichen Produktionsperiode $T$ und der volkswirtschaftlichen Bo-

Prof. Dr. Carl Christian von Weizsäcker ist emeritierter Professor für Volkswirtschaftslehre der Universität zu Köln und Senior Research Fellow des MaxPlanck-Instituts zur Erforschung von Gemeinschaftsgütern in Bonn.

Prof. Dr. Hagen Krämer ist Professor für Volkswirtschaftslehre an der Fakultät für Wirtschaftswissenschaften der Hochschule Karlsruhe - Technik und Wirtschaft. 
Abbildung 1

Begrenzte Mehrergiebigkeit der Produktionsumwege

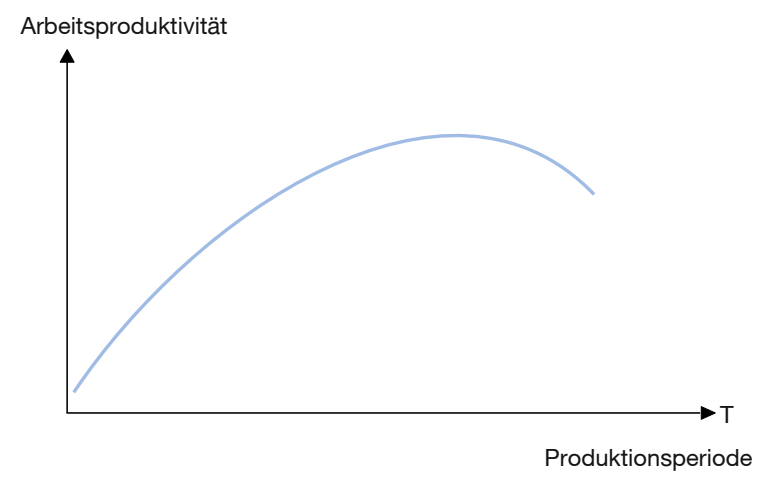

Quelle: eigene Darstellung.
Abbildung 2

Das Spardreieck

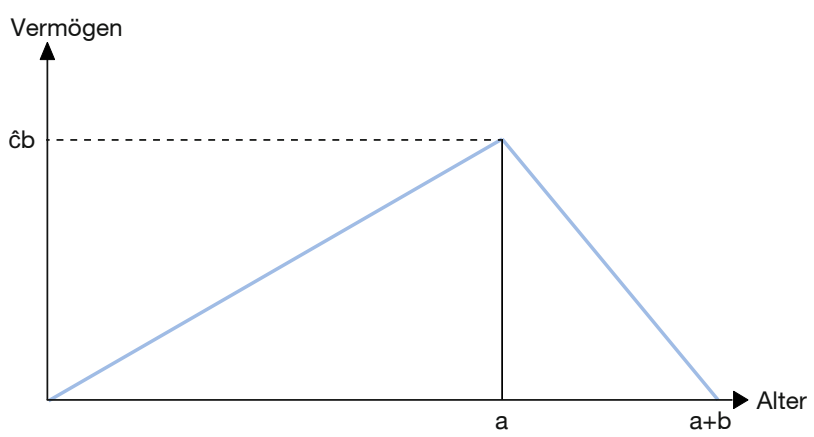

Es bedeuten: a: Erwerbsphase (in Jahren), b: Ruhestandsphase (in Jahren), c: Konsum in Geldeinheiten pro Jahr, $Z$ = ĉb/2: durchschnittlicher relativer Vermögenswunsch in Geldeinheiten.

Quelle: v. Weizsäcker und Krämer (2019), 46.

Eine der zentralen Ursachen für die Grenze der Mehrergiebigkeit höherer Komplexität ist der technische Fortschritt. Durch den kontinuierlichen Wandel der Wissensgrundlagen veralten die Zwischenprodukte, die den Realkapitalstock bilden. Wenn sie „zu alt“ werden, müssen sie ersetzt werden. Daher gibt es Grenzen für die nützliche Kapitalbindung des Produktionsprozesses. Hiervon rührt empirisch die säkulare Konstanz des Verhältnisses von Kapitaleinsatz und Produktionsergebnis und damit der Produktionsperiode. Die seit längerem niedrigen realen Zinssätze in praktisch allen entwickelten Ländern legen nahe, dass die von uns betrachteten Volkswirtschaften der OECD-Länder und Chinas schon nahe an dem Produktionsumweg sind, der die Ergiebigkeit der ursprünglichen Produktionsfaktoren Arbeit und Boden maximiert. genz. Eine Ursache ist die Grenze für die „Mehrergiebigkeit längerer Produktionsumwege“, die man auch als „Grenze der Mehrergiebigkeit höherer Komplexität" bezeichnen kann. Böhm-Bawerks Gesetz gilt nicht universell, sondern nur bis zu einem gewissen Punkt. Das liegt an den überproportional steigenden Kosten der Komplexität. Je weiter ausgreifend die Produktionsumwege sind, desto komplexer wird das Produktionssystem, was sich in einer ab einem bestimmten Punkt sinkenden Arbeitsproduktivität niederschlägt (vgl. Abbildung 1). Sie ergibt sich, wenn eine Überkomplexität bzw. eine Überspezialisierung der Wirtschaft auftritt. Dann wird die BruttoMehrergiebigkeit eines höheren $T$ von den Mehrkosten höherer Komplexität ganz aufgefressen: auch bei einem Zinssatz von null und bei Tilgungsraten von null wird sich der Facharbeiter kein Schloss zum Wohnen leisten. Die Instandhaltungskosten würden inn ins Elend bringen. Und der Manager kennt den Spruch: „KISS: keep it simple, stupid“".
Die andere Ursache betrifft das wachsende Kapitalangebot, das ebenfalls auf den Wandel oder Fortschritt des Wissens zurückgeführt werden kann. Es ist das von uns formulierte "Gesetz der mit dem Wohlstand steigenden Zukunftsorientierung" menschlichen Verhaltens. Je höher der Wohlstand, desto höher ist der relative Vermögenswunsch. Das Verhältnis zwischen dem geplanten Vermögen und dem laufenden Konsum (der sogenannten Vermögenskoeffizient) steigt an. Das "Gesetz des wachsenden Vermögenskoeffizienten" ist dasselbe wie das „Gesetz der wachsenden privaten Warteperiode Z“. Der steigenden privaten Warteperiode entspricht die mit dem Wohlstand steigende Lebenserwartung. Denn nirgends in der Welt gibt es eine hierzu parallel steigende Lebensarbeitszeit. Daher steigt die Rentenbezugsdauer. Parallel zu ihr steigt die Sparquote während der Berufstätigkeit. Das Spardreieck bringt diesen Zusammenhang als grafische Darstellung zum Ausdruck (vgl. Abbildung 2). Trotz der 
Tabelle 1

Zusammensetzung des privaten Vermögens in der Region OECD plus China

Werte in Jahren volkswirtschaftlichen Konsums (2015)

\begin{tabular}{lcccc} 
& \multicolumn{2}{c}{ bei positivem Realzins } & bei Nullzins \\
& $\begin{array}{c}\text { Deutsch- } \\
\text { land }\end{array}$ & OECD & $\begin{array}{c}\text { OECD + } \\
\text { China }\end{array}$ & $\begin{array}{c}\text { OECD + } \\
\text { China }\end{array}$ \\
\hline Realkapital & 2,76 & 3,59 & 4,03 & 4,51 \\
\hline Boden & 1,54 & 2,02 & 1,94 & 2,52 \\
\hline Staatsschulden & 5,03 & 4,27 & 3,92 & 6,14 \\
\hline -explizit & 0,70 & 0,92 & 0,75 & 0,75 \\
\hline -implizit (GRV) & 3,64 & 2,71 & 2,60 & 4,82 \\
\hline -implizit (GKV und SPV) & 0,69 & 0,64 & 0,57 & 0,57 \\
\hline Summe & 9,33 & 9,88 & 9,89 & 13,17 \\
\hline
\end{tabular}

Anmerkungen: explizite Staatsschulden werden als Netto-Staatsschulden ausgewiesen (zur Definition v. Weizsäcker und Krämer, 2019, 162 f.), implizite Staatsschulden stellen Forderungen der Versicherten gegenüber den Sozialversicherungen dar (zur Definition v. Weizsäcker und Krämer, 2019, 168-199).

Abkürzungen: GRV: Gesetzliche Rentenversicherung, GKV: Gesetzliche Krankenversicherung, SPV: Soziale Pflegeversicherung.

Quelle: eigene Darstellung; v. Weizsäcker und Krämer (2019).

darin liegenden Vereinfachung gibt das Spardreieck die Verhältnisse z. B. für Deutschlands Arbeitnehmer approximativ wieder. Dabei muss man die aus der gesetzlichen Rentenversicherung resultierenden Rentenanwartschaften ebenfalls als Teil der Sparleistungen ansehen.

Wir haben für das Jahr 2015 eine empirische Abschätzung des Nettovermögens der Bürger in Deutschland sowie in den übrigen OECD-Ländern und für China vorgenommen (vgl. Tabelle 1). Alle Daten für unsere Berechnungen stammen entweder direkt oder indirekt aus Quellen der amtlichen Statistik. Um ein Beispiel zu nennen: Zur Ermittlung der Ansprüche der EU-Bürger gegenüber den gesetzlichen Rentenversicherungen konnten wir auf Daten von Eurostat über die Höhe der Renten- und Pensionsanwartschaften in insgesamt 29 Ländern zurückgreifen. Für die restlichen Länder wurden länderspezifische amtliche Quellen herangezogen oder Schätzungen vorgenommen.

Die einzelnen Bestandteile des privaten Vermögens - Realkapital, Boden und Staatsschulden - setzen wir jeweils ins Verhältnis zum Wert des volkswirtschaftlichen Konsums eines Landes. In Deutschland machten 2015 die Ansprüche der privaten Bürger gegenüber dem Staat mit 5,03 Jahren volkswirtschaftlichen Konsums den größten Bestandteil des privaten Vermögens aus (Realkapital: 2,76; Boden: 1,54). Für den OECD-Raum plus China war die Vermögenskomponente Realkapital mit 4,03 Jahren
Abbildung 3

Die drei Formen privaten Vermögens und ihre Anteile

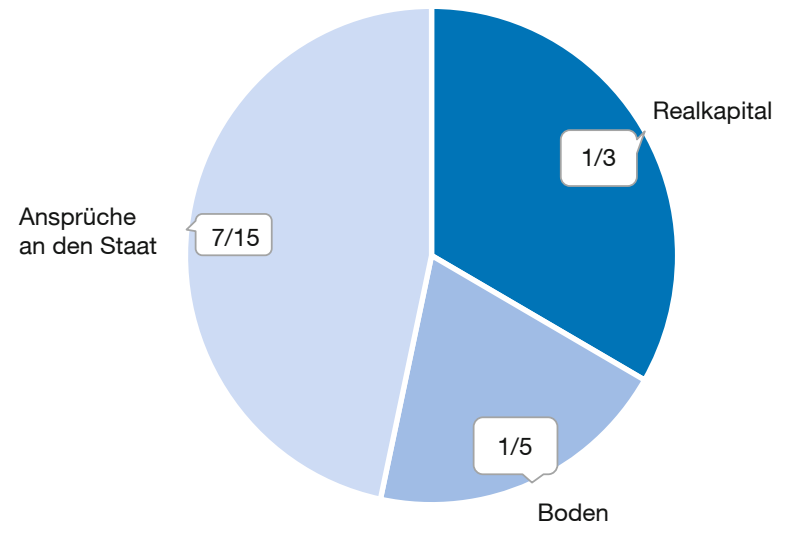

Quelle: v. Weizsäcker und Krämer (2019), 3.

volkswirtschaftlichen Konsums etwas größer als die Kategorie Staatsschulden mit 3,92 Jahren volkswirtschaftlichen Konsums. Insgesamt betrug das Vermögen des privaten Sektors im OECD-Raum plus China ungefähr zehn Jahre volkswirtschaftlichen Konsums.

Diese Werte wurden unter der Annahme eines positiven Realzinssatzes ermittelt. Im Zusammenhang mit unserer These vom negativen natürlichen Zins waren wir speziell an der Situation eines Realzinses von null interessiert. Daher haben wir für die Eurostat-Länder durch eine Extrapolation den Barwert der Verbindlichkeiten aus der Rentenversicherung auf Basis eines Nominalzinses von $2 \%$ bzw. eines Realzinses von $0 \%$ berechnet. Auf Grundlage der hierdurch gewonnenen Ergebnisse konnten wir auch für die übrigen Länder Schätzungen von Anwartschaftsbarwerten aus den Renten- und Pensionssystemen vornehmen. Wir schätzen, dass bei einem realen Diskontierungssatz von null das explizite und das implizite Nettovermögen der Bürger gegenüber dem Staat in der Region OECD plus China rund sechsmal so hoch ist wie der jährliche volkswirtschaftliche Konsum. Auch für die Vermögensbestandteile Realkapital und Boden haben wir eine spezielle Methode entwickelt, um die jeweiligen Vermögenswerte bei einem Realzins von null abzuschätzen.

Wir kommen im Endergebnis dazu, dass das gesamte Nettovermögen des privaten Sektors in der Region OECD plus China im Jahr 2015 mehr als das 13-fache des jährlichen volkswirtschaftlichen Konsums betrug, also $Z=13$ war. Ein Drittel davon machte $T$ aus, bestand somit aus Vermögen in Form von Realkapital (Maschinen, Anlagen, Gebäude); ein Fünftel war $L$, bestand also aus Boden; und knapp die Hälfte (genauer: 7/15) war $D$, also finanzielle Netto-Ansprüche gegenüber dem Staat (vgl. Abbildung 3). 
Wir argumentieren in unserem Buch, dass der natürliche Zins negativ ist. Dieser ist der hypothetische reale Vollbeschäftigungszins, der gelten würde, wenn die Bürger keine positive Nettoposition gegenüber dem Staat hätten. Bei einem Realzins von null, und wenn es keine Staatsschulden geben würde, gäbe es einen riesigen Überschuss des Kapitalangebots gegenüber der Kapitalnachfrage. Dieser Überschuss drückte auf die Beschäftigung und/oder die Kapitalrendite, die damit ins Negative absinken würde. Weil aber der Nominalzins eine untere Schranke hat, kann der Realzins nur negativ werden, wenn es starke Inflation gibt. Wenn wir gemäß unseren Untersuchungen zu dem Schluss kommen, dass beim gegebenen Steuersystem oder bei einem optimalen Steuersystem der natürliche Zins negativ ist, so hat die Gesellschaft die Wahl zwischen einem negativen Realzins bei Inflation ohne Staatsschulden und einem Zustand der Preisstabilität mit positiven Staatsschulden.

Staatsschulden werden häufig als ein zu vermeidendes Übel angesehen. Diese Sichtweise hat in Deutschland dazu beigetragen, dass 2009 eine "Schuldenbremse“ ins Grundgesetz aufgenommen wurde. Diese wurde im Frühsommer 2020 angesichts der Corona-Pandemie kurzfristig ausgesetzt, um dem Staat eine größere Neu- verschuldung zu ermöglichen. Der nicht nur in Deutschland notwendige sprunghafte Anstieg der Staatsverschuldung stellt aus unserer Sicht jedoch kein Problem dar - im Gegenteil. Denn generell gilt, dass Staatsschulden immer auch privates Vermögen sind. Sie sind für die öffentliche Hand tragfähig, wenn deren Verzinsung nicht höher liegt als die Wachstumsrate der Volkswirtschaft. Diese Bedingung ist dann erfüllt, wenn es der Staat mit der Staatsverschuldung nicht übertreibt. Aufgrund der großen Divergenz zwischen Sparen und Investieren im 21. Jahrhundert ist es dringend geboten, die gegenwärtige „Schuldenbremse" wieder abzuschaffen und durch besser geeignete Instrumente zu ersetzen. Wir schlagen vor, stattdessen ein internationales Bilanzabkommen zu vereinbaren, mit dem auch die globalen Leistungsbilanzungleichgewichte wieder abgebaut werden können.

\section{Literatur}

Smith, A. (1776): An Inquiry into the Nature and Causes of the Wealth of Nations.

v. Böhm-Bawerk, E. (1889), Kapital und Kapitalzins, Zweite Abteilung, Positive Theorie des Kapitales.

v. Weizsäcker, C. C. und H. Krämer (2019): Sparen und Investieren im 21. Jahrhundert. Die große Divergenz, Springer. 